\title{
High prevalence of Campylobacter jejuni CC21 and CC257 clonal complexes in children with gastroenteritis in Tehran, Iran
}

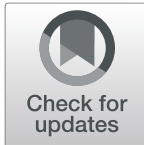

Mahnaz Sarhangi, Bita Bakhshi* [D and Shahin Najar Peeraeyeh

\begin{abstract}
Background: Campylobacter jejuni (C. jejuni) is a leading cause of acute gastroenteritis in human worldwide. The aim of study was to assess the distribution of sialylated lipooligosaccharide (LOS) classes and capsular genotypes in C. jejuni isolated from Iranian children with gastroenteritis. Furthermore, the level of dnaK gene expression in C. jejuni strains with selected capsular genotypes and LOS classes was intended. Moreover, a comprehensive study of C. jejuni MLST-genotypes and inclusive comparison with peer sequences worldwide was intended.
\end{abstract}

Methods: Twenty clinical C. jejuni strains were isolated from fecal specimens of 280 children aged 0-5 years, suspected of bacterial gastroenteritis, which admitted to 3 children hospitals from May to October, 2018. Distribution of sialylated LOS classes and specific capsular genotypes were investigated in C. jejuni of clinical origin. The expression of dnaK in C. jejuni strains was measured by Real-Time-PCR. MLST-genotyping was performed to investigate the clonal relationship of clinical C. jejuni strains and comparison with inclusive sequences worldwide.

Results: C. jejuni HS23/36c was the predominant genotype (45\%), followed by HS2 (20\%), and HS19 and HS4 (each $10 \%)$. A total of $80 \%$ of isolates were assigned to LOS class B and C. Higher expression level of dnaK gene was detected in strains with HS23/36c, HS2 and HS4 capsular genotypes and sialylated LOS classes B or C. MLST analysis showed that isolates were highly diverse and represented 6 different sequence types (STs) and 3 clonal complexes (CCs). CC21 and CC257 were the most dominant CCs (75\%) among our C. jejuni strains. No new ST and no common ST with our neighbor countries was detected.

Conclusions: The C. jejuni isolates with LOS class B or C, and capsular genotypes of HS23/36, HS2, HS4 and HS19 were dominant in population under study. The CC21 and CC257 were the largest CCs among our isolates. In overall picture, CC21 and CC353 complexes were the most frequently and widely distributed clonal complexes worldwide, although members of CC353 were not detected in our isolates. This provides a universal picture of movement of dominant Campylobacter strains worldwide.

Keywords: C. Jejuni, LOS class, Capsular genotype, dnaK, MLST, Iran

\footnotetext{
* Correspondence: b.bakhshi@modares.ac.ir

Department of Bacteriology, Faculty of Medical Sciences, Tarbiat Modares

University, Jalal-Ale-Ahmad Ave, Tehran 14117-13116, Iran
}

(c) The Author(s). 2021 Open Access This article is licensed under a Creative Commons Attribution 4.0 International License, which permits use, sharing, adaptation, distribution and reproduction in any medium or format, as long as you give appropriate credit to the original author(s) and the source, provide a link to the Creative Commons licence, and indicate if changes were made. The images or other third party material in this article are included in the article's Creative Commons licence, unless indicated otherwise in a credit line to the material. If material is not included in the article's Creative Commons licence and your intended use is not permitted by statutory regulation or exceeds the permitted use, you will need to obtain permission directly from the copyright holder. To view a copy of this licence, visit http://creativecommons.org/licenses/by/4.0/ The Creative Commons Public Domain Dedication waiver (http://creativecommons.org/publicdomain/zero/1.0/) applies to the data made available in this article, unless otherwise stated in a credit line to the data. 


\section{Introduction}

Campylobacter jejuni (C. jejuni) is a leading cause of bacterial foodborne poisoning and acute gastroenteritis in human worldwide [1]. This bacterium often causes a moderate to severe watery regularly self-limiting gastroenteritis and post-infectious immune disorders such as Guillain-Barre syndrome (GBS) [2].

C. jejuni produces Capsular Polysaccharide (CPS). The CPS gene cluster is located in a hypervariable region in the $C$. jejuni genome [3]. Major serodeterminant of the CPS is in classical Penner or Heat-Stable (HS) serotyping Scheme. In the classical Penner serotyping scheme, which is based on the passive slide hemagglutination assay, C. jejuni strains are divided into 47 serotypes, of which, due to similarity in the CPS structure, 35 serotypes have been refined, which are serotype crossreactive pairs or complexes $[4,5]$. C. jejuni Penner serotypes associated with GBS often belong to HS1, HS4c, HS19, HS23/36c and HS41, furthermore, the most common serotypes among sporadic cases are reported in the HS4c, HS2 and HS1 [4]. Recently, CPS genotyping was used as a more effective method, since it is not affected by variations in capsular gene expression which is observed in serotyping [2]. Moreover, CPS genotyping is a fast, accessible and reliable method to determine CPS types in C. jejuni [2, 3].

The cluster of genes involved in $C$. jejuni lipooligosaccharide (LOS) biosynthesis, is one of the most variable regions of $C$. jejuni genome [6] due to mutations within or recombination between LOS biosynthesis gene/gene regions [7].

Among 19 different LOS locus classes from A to S, 3 classes (A, B and C) play a key role in the biosynthesis of the sialic acid and are often isolated from the stools of patients with GBS $[2,6,8]$.

Post-infection diseases like GBS with $C$. jejuni have been proved to be associated with antibodies of human gangliosides. The induction of these autoantibodies is associated with molecular mimicry between human gangliosides and bacterial epitopes present at the surface of the LOS [9]. It is worth noting that the antibody responses to gastroenteritis is different to GBS triggered by $C$. jejuni [10]. In addition to antiganglioside antibodies, Heat Shock Proteins (HSP) family can mediate in the autoimmune diseases. They belong to a highly protected family that is present in normal physiological conditions in prokaryotic and eukaryotic cells. These proteins are etiologic factors in many autoimmune diseases in such a way that their overexpression leads to environmental stress induction [9, 11].

C. jejuni carry several of HSPs, including groELS, dnaJ, dnaK and lon [12] among which DnaK proteins (70 kDa) has a high sequence homology with HSP70 of human peripheral neurons [9]. A high titer of anti-HSP antibody (HSP27, HSP60 and HSP70) can be found in CSF (Cerebrospinal fluid) of patients with GBS [9].

To date, no study has been reported on the distribution of CPS genotypes, LOS locus classes and Multilocus sequence typing (MLST) of C. jejuni in Iran. The aim of our study was to assess, for the first time, the distribution of sialylated LOS classes and capsular genotypes among clinical $C$. jejuni strains isolated from Iranian children with gastroenteritis. Moreover, the correlation of DnaK protein expression level in C. jejuni strains with selected capsular genotypes and LOS classes was intended. Furthermore, a comprehensive comparison of C. jejuni MLST genotypes with peer sequences reported worldwide was envisioned.

\section{Materials and methods \\ Phenotypic and genotypic identification of $C$. jejuni strains from fecal samples}

Based on Cochran formula for calculating of sample size, a total of 3000 gastroenteritis cases were examined for suspected cases of sporadic campylobacteriosis. Gastroenteritis was characterized as abdominal pain with $\geq 3$ episodes per day. Children with underlying gastrointestinal disease, physiologic diarrhea or history of antibiotic intake were excluded, which were defined by physicians in hospitals. Suspected cases of bacterial gastroenteritis were subjected to specimen collection, among which 280 cases accompanied WBC (white blood cell) and RBC (red blood cell) shedding in the majority of cases and were considered as suspected cases of campylobacteriosis.

Fecal specimens were collected from children with gastroenteritis, aged $0-5$ years, referred to 3 Children's Medical Center and Hospitals at Tehran, Iran, from May to October 2018. Information on age, clinical symptoms, history of non-pasteurized dairy products consumption, animal contact as well as laboratory results was recorded. Then specimens were transferred from laboratory of hospital to the laboratory of Tarbiat Modares University using Carry-Blair Transport Media (Micro Media-Hungary) and immediately streaked on Brucella agar and modified charcoal-cefoperazone-deoxycholate agar (mCCDA) (Merck-Germany). Plates were incubated at $42{ }^{\circ} \mathrm{C}$ for $48 \mathrm{~h}$ under microaerophilic condition using Gas Pack C (Merck-Germany). Gram staining, spiral morphology, catalase and oxidase production, nitrate reduction and indoxyl acetate hydrolysis test were used to confirm C. jejuni colonies. Also, hippurate hydrolysis test was used for phenotypic distinguishing of $C$. jejuni from C. coli from enteritis patients. Eventually, twenty C. jejuni and three C. coli strains were confirmed by Duplex PCR [13] and in the following, twenty $C$. jejuni was studied. 
Table 1 The primer sequences included in the C. jejuni capsule typing scheme

\begin{tabular}{|c|c|c|c|c|}
\hline Primer & Forward sequence & Reverse sequence & Product size (bp) & Reference \\
\hline HS1 & GCAAGAGAAACATCTCGCCTA & TTGGCGGTAAGTIITGAAGA & 610 & [5] \\
\hline HS2 & CATCCTAGCACAACTCACTTCA & CAGCATTGGAGGATTTACAATATAT & 62 & {$[5]$} \\
\hline HS4A & CCTAACATATCATACACTACGGT & TATATTTGGTTAGGGATCCA & 370 & [5] \\
\hline HS19 & GGCAACAAACAAACATATTCAGA & CGAGGATGAAAATGCCTCAA & 450 & {$[5]$} \\
\hline HS23/36 & GCTITATATCTATCCAGTCCATTATCA & GCTTGGGAGATGAATTTACCTTIA & 161 & {$[5]$} \\
\hline HS41 & TGCAATCTCTAAAGCCCAAG & CTTACATATGCTGGTAGAGATGATATG & 279 & {$[5]$} \\
\hline
\end{tabular}

Twenty-three Campylobacter isolates were identified from 280 stool samples among which 20 and 3 isolates were $C$. jejuni and $C$. coli, respectively. All confirmed $C$. jejuni strains $(n=20)$ were designated and used for further analyses.

\section{Identification of capsular genotypes and LOS locus classes}

The capsular genotypes of $C$. jejuni strains were identified using specific primers for most commonly found genotypes HS1, HS2, HS4c, HS19, HS23/36c and HS41 (Table 1) $[4,5]$.

From 19 different LOS locus classes from A to S, 3 classes (A, B and C) play a key role in the biosynthesis of the sialic acid and are often found in isolates from the stools of patients with GBS [2, 6, 8]. Therefore, sets of primers specific for class $A, B$, and $C$ classes were used for characterization of LOS identities (Table 2) [6].

Genomic DNA was extracted using a genomic DNA extraction kit (GeNetBio, Korea), according to the manufacturer instructions. PCRs was carried out within a thermal cycler (Eppendorf, Germany) in a final volume of $25 \mu \mathrm{L}$ containing 1-10 ng DNA template, $2.5 \mu \mathrm{L} 10 \mathrm{X}$ PCR buffer, 1 unit of Taq DNA polymerase, $2.0 \mathrm{mM} \mathrm{MgCl}, 0.2 \mu \mathrm{M}$ of each primer, $0.3 \mathrm{mM}$ each $\mathrm{dNTP}$ and sterile deionized water. Amplification conditions was as follow: $95^{\circ} \mathrm{C}$ for 5 min, followed by 30 amplification cycles including denaturation at $94{ }^{\circ} \mathrm{C}$ for $1 \mathrm{~min}$, annealing at $52^{\circ} \mathrm{C}$ for $1 \mathrm{~min}$ and extension at $72{ }^{\circ} \mathrm{C}$ for $1 \mathrm{~min}$. The reaction was ended with a final extension at $72{ }^{\circ} \mathrm{C}$ for $5 \mathrm{~min}$ and followed by electrophoresis of amplicons on $1 \%$ agarose gel.
Real-time PCR for dnaK gene expression in clinical $C$. jejuni isolates

Microbial DnaK is a bacterial conserved chaperone protein which has a sequence homology with human peripheral nerve HSP70 and its high level expression is supposed to be related to GBS promotion [14, 15].

In order to assess the efficiency of real-time PCR amplification, five serial 1:10 dilutions of cDNA was used as template for qRT-PCR reaction of the dnaK and 16srRNA genes. The CTs values and the concentrations of the template were used to plot the standard curve and calculate the primer efficiency.

RNA extraction was performed on 20 C. jejuni strains, which were previously checked for the presence of capsular types and LOS locus classes using a Favoren Biotec Corp kit (Taiwan). Subsequently, the RNA molecules were treated using the DNase I kit (TaKaRa). A cDNA synthesis kit (Yekta Tajhiz Azma-Iran) was used to generate a single-strand cDNA. The cDNAs were kept at $20^{\circ} \mathrm{C}$. Quantitative Real Time-PCR was performed using SYBR Green (RealQ Plus Master Mix Green-Denmark) in Qiagen-Rotor-Gene Q with HRM. 16S rRNA gene was used as the internal control. One of the isolates that neither had the selected capsular serotypes nor the LOS locus classes was considered as a reference gene. The PCR reaction mixture consisted of $100 \mathrm{ng}$ to $1 \mathrm{mg}$ of cDNA (for dnaK and 16S rRNA genes), $1 \mathrm{mM}$ of each primer (Table 3) [16] and $12.5 \mathrm{~mL}$ of SYBR Green I Master Mix. Cycling conditions included an initial denaturing step of $10 \mathrm{~min}$ at $95^{\circ} \mathrm{C}$ followed by 40 cycles of $15 \mathrm{~s}$ at $95^{\circ} \mathrm{C}$ and $1 \mathrm{~min}$ at $60^{\circ} \mathrm{C} .2^{-\Delta \Delta C t}$ is a relative quantification method for analyze the relative changes in gene

Table 2 Primer used for identification of classes A, B and C

\begin{tabular}{|c|c|c|c|c|}
\hline ORF & Forward sequence & Reverse sequence & Product size (bp) & Reference \\
\hline Orf7ab & ACTACACTTTAAAACATTTAATCC AAAATCA & CCATAAGCCTCACTAGAAGGTATGAGTATA & 580 & [6] \\
\hline Orf 6ab1 & CAAGGGCAATAGAAAGCTGTATCA & ACAAGCACTTCATTCTTAGTATTACAAAT & 631 & [6] \\
\hline Orf6ab2 & TCATCTTGCCAACTTATAATGTGGA & TCTAGCGATATTAAACCAACAGCCT & 517 & [6] \\
\hline Orf5bll & CTGTGATGATGGGAGTGAAGAGC & GGTAATCGTTTCGGCGGTATT & 539 & [6] \\
\hline Orf6c & GTAGTAGATGATTGTGGTAATGATAAA & ATAGAATTGCTATTTACATGCTGG & 554 & [6] \\
\hline Orf7c & TTGAAGATAGATATTTTGTGGGTAAA & CTITAAGTAGTGTITTATGTCACTTGG & 746 & [6] \\
\hline
\end{tabular}


Table 3 Specific primers used for Real-Time PCR

\begin{tabular}{lllll}
\hline Primer & Forward sequence & Reverse sequence & Product size (bp) & Reference \\
\hline dnaK & AAACGCCAAGCGGTAACTAA & TTCTTAGCCGCGTCTTCAT & 90 & {$[16]$} \\
16SrRNA & AAGGGCCATGATGACTTGACG & AGCGCAACCCACGTATTAG & 107 & {$[16]$}
\end{tabular}

expression from real-time quantitative PCR [16]. SPSS software version 20 was used for the analysis of data.

\section{Multilocus sequence typing (MLST)}

The MLST method was performed according to Dingle, et al. [17]. Each $25 \mu \mathrm{L}$ amplification reaction mixture comprised 1-10 ng DNA template, $1 \mathrm{X}$ PCR buffer, 1.25 unit of Taq DNA polymerase, $1.5 \mathrm{mM} \mathrm{MgCl}, 1 \mu \mathrm{M}$ of each primer (Table 4), $0.8 \mathrm{mM}$ each $\mathrm{dNTP}$ and sterile deionized water.

The PCR conditions were as follows: denaturation at $94{ }^{\circ} \mathrm{C}$ for $2 \mathrm{~min}$; annealing at $50{ }^{\circ} \mathrm{C}$ for $1 \mathrm{~min}$, extension at $72{ }^{\circ} \mathrm{C}$ for $1 \mathrm{~min}$ for 35 cycles. DNA Sequences of each housekeeping gene were submitted to C. jejuni MLST database (http://pubmlst.org/campylobacter) and the related allelic numbers, Sequence Types (ST) and Clonal Complexes (CC) were identified [18]. The Accession Numbers of DNA Sequences of 20 C. jejuni isolates were deposited in GenBank (https://pubmlst.org/ campylobacter). Dendrogram was plotted using Interactive Tree Of Life (iTOL) v4 [19].

\section{Geographic distribution of STs and CCs}

A circular dendrogram was plotted for comparison of the peer sequences reported worldwide. A total of 72, 392 isolates were downloaded from PubMLST website and analyzed [18]. Based on the inclusion and exclusion criteria, a total of 304 isolates were included in dendrogram. The inclusion criteria were: $C$. jejuni strains from the five recent years (2014-2018), C. jejuni strains from human stool, $C$. jejuni strains from the sporadic cases and gastroenteritis. The inclusion criteria were based on parameters that well warrant comparisons with our strains. Excluding criteria were unspecified ST or CC as well as repeated ST samples of each country.
The recorded MLST data in PubMLST database were also used to compare STs and CCs between our isolates with those from our neighbor countries. Among Iran neighboring countries only Turkey and Pakistan had recorded data about C. jejuni in PubMLST. Eleven isolates from human stool samples has been reported form these countries which were included for the final analysis. Dendrograms was plotted using Interactive Tree Of Life (iTOL) v4 [19]. Various sequence types were obtained from and plotted using PubMLST database tools [18].

\section{Statistical analysis}

To assess the presence of sialylated LOS classes A, B and $\mathrm{C}$ and CPS types in $20 \mathrm{C}$. jejuni strains isolated from children with gastroenteritis, we used the Cochran's Q test. Data were analyzed with the Statistical Package for Social Sciences (Version 25.0, SPSS Inc., Chicago, IL, USA); $p<0.05$ was considered statistically significant.

\section{Results}

Twenty-three Campylobacter isolates were identified from 280 stool samples of children with gastroenteritis. Among the 23 isolates, 20 and 3 isolates were $C$. jejuni and $C$. coli, respectively, based on hippurate hydrolysis test and duplex PCR assay of $c a d F$ gene. Analysis of the duplex PCR assay of $c a d F$ gene showed that 737 and 461 bp amplicons were corresponding to $C$. jejuni and $C$. coli, respectively. All confirmed $C$. jejuni strains $(n=20)$ were designated and used for further analyses.

\section{CPS genotype and LOS locus class diversity}

From 20 C. jejuni isolates, 17 (85\%) expressed one of the selected CPS genotypes under study. CPS types HS23/36c were found in 9 isolates (45\%) and appeared as dominant CPS among 20 C. jejuni strains (sig = 0.001; Cochran's Q

Table 4 Primers used for Campylobacter jejuni MLST

\begin{tabular}{|c|c|c|c|c|}
\hline Primer & Forward & Reverse & Product size (bp) & Reference \\
\hline asp & CCAACTGCAAGATGCTGTACC & TTAATITGCGGTAATACCATC & 625 & [17] \\
\hline$g \ln$ & CATGCAATCAATGAAGAAAC & TTCCATAAGCTCATATGAAC & 722 & [17] \\
\hline$g / t$ & GTGGCTATCCTATAGAGTGGC & CCAAAGCGCACCAATACCTG & 575 & [17] \\
\hline gly & AGCTAATCAAGGTGTTTATGCGG & AGGTGATTATCCGTTCCATCGC & 648 & [17] \\
\hline pgm & GGTITTAGATGTGGCTCATG & TCCAGAATAGCGAAATAAGG & 700 & {$[17]$} \\
\hline$t k t$ & GCTTAGCAGATATTTTAAGTG & ACTTCTTCACCCAAAGGTGCG & 691 & {$[17]$} \\
\hline unc & TGTTGCAATTGGTCAAAAGC & TGCCTCATCTAAATCACTAGC & 631 & [17] \\
\hline
\end{tabular}


test). HS2, HS19, and HS4 were detected in 4 (20\%), 2 $(10 \%)$ and $2(10 \%)$ isolates, respectively. No C. jejuni isolate belonged to HS1 or HS41 genotypes.

Of 20 strains, 16 (80\%) expressed sialylated LOS locus class B or C. The LOS class B with 11 isolates (55\%) was dominant sialylatd LOS class ( $\mathrm{sig}=0.003$; Cochran's Q test). No isolate with LOS class A was found among our isolates.

\section{Relationship between CPS type and LOS locus class}

Among $C$. jejuni strains with LOS class B, the distribution of CPS types was as follows: HS23/36c $(n=6,54$ / $54 \%)$, HS2 $(n=2,18 / 18 \%)$, HS4 $(n=2,18 / 18 \%)$ and HS19 ( $n=1,9 / 9 \%)$. Also, the distribution of CPS genotypes among $C$. jejuni strains with LOS class $\mathrm{C}$ was as HS23/36c $(n=2,40 \%)$ and HS2 ( $n=2,40 \%)$ (Fig. 1$)$.

\section{Comparison of dnaK gene expression in clinical C. jejuni strains}

The primer efficiency was calculated as $97.51 \%$ and 103.54 for dnaK and 16srRNA genes, respectively. The dnaK gene expression was determined by Quantitative Real-Time PCR and according to the $2^{-\triangle \Delta C T}$ method. Among isolates that showed one of the LOS classes of A-C or one of six selected capsular genotypes, 18 isolates were classified into three groups including group 1 : with identified CPS genotype and sialylated LOS class (B or C) $(n=15)$, group 2: with identified CPS genotype and without sialylated LOS class $(n=2)$, group 3: without CPS genotype but with sialylated LOS class $(n=1)$, and one of the isolates that neither had the selected capsular serotypes nor the LOS locus classes was considered as a reference strain. Due to insufficiency of data, differential expression analysis could not be performed; thus, descriptive statistics was used instead. As a result, dnaK expression level in group 1 was greater than other groups (Table 5). The dnaK expression level was much higher in clinical $C$. jejuni isolates with one of the CPS genotypes and the LOS classes relevant to GBS patients. Based on the $2^{-\Delta \Delta C T}$ method, the graph of the fold change of dnaK gene expression was plotted (Fig. 2).

\section{CC and ST variation of MLST analysis}

Based on the MLST analysis of 20 isolates, 6 sequence types and 3 clonal complexes were detected in Iran. Five and 7 isolates were identified as ST-257 and ST-50, respectively. Each of the ST-19 and ST-5326 were detected in three isolates, while ST-1096 and ST-1113, each were only detected in one isolate.

Out of the 3 identified clonal complexes, ST-21 complex dominated in 10 isolates (50\%).

Both ST-257 complex and ST-828 complex were found in $5(25 \%)$ and $2(10 \%)$ isolates, respectively.

Distribution of the sequence types and the genetic link between C. jejuni strains isolated from patients with gastroenteritis has been shown in the dendrogram (Fig. 3). The relationship among 20 isolates based on clonal complexes is reflected in minimum spanning tree diagram (Fig. 4) [20].

\section{Linkage between CPS genotypes and LOS class with MLST CC}

Totally, 3 CCs (CC828, CC257 and CC21) were identified. The isolates with HS23/36c and HS19 genotypes were found in CC21 (Fig. 5). Majority of isolates in CC21 had LOS class B or LOS class C. In CC257, 4 isolates belonged to HS2 serotype. In this CC, LOS class C and $\mathrm{B}$ were observed. Two isolates were assigned to CC828 (Fig. 6).

\section{Geographic distribution of STs and CCs}

A phylogenetic analysis revealed that there was no common STs or CCs between Iran and its neighbor countries, Turkey and Pakistan (Fig. 7). This may be due to scarcity of published data from these countries. No data is available from other neighboring countries of Iran.

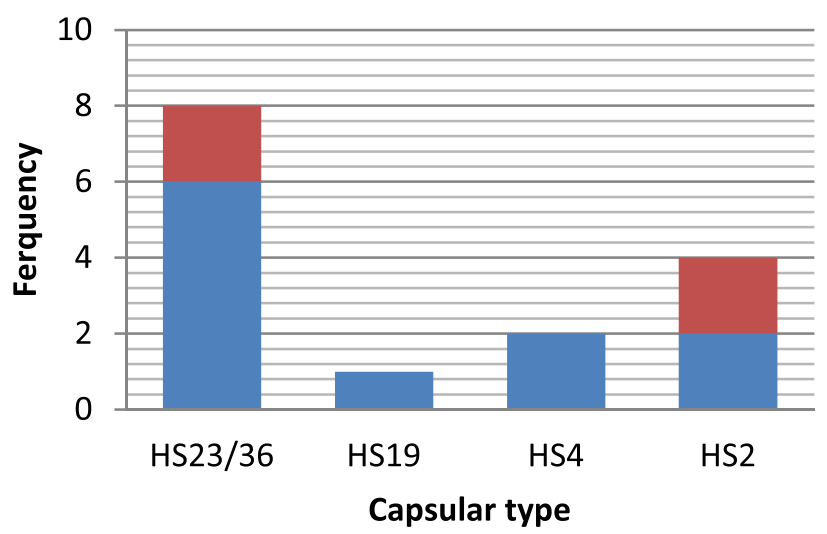

Calss C

Calss B 
Table 5 Relationship between the level of dnaK gene expression in clinical C. jejuni isolates

\begin{tabular}{|c|c|c|c|c|}
\hline Group & Isolate ID & Capsular genotype & LOS class & Fold Change: $2^{-\Delta \Delta C T}$ \\
\hline G1 & 79,434 & HS2 & $B$ & $2.04 \pm 0.72$ \\
\hline G1 & 79,425 & HS23/36 & B & $1.54 \pm 0.44$ \\
\hline G1 & 79,426 & HS23/36 & B & $1.34 \pm 0.3$ \\
\hline G1 & 79,438 & HS23/36 & B & $5.43 \pm 1.7$ \\
\hline G1 & 79,420 & HS2 & C & $2.66 \pm 0.99$ \\
\hline G1 & 79,437 & HS23/36 & $B$ & $3.0 \pm 1.1$ \\
\hline G1 & 79,427 & HS4 & B & $2.16 \pm 0.78$ \\
\hline G1 & 79,435 & HS2 & B & $2 \pm 0.7$ \\
\hline G1 & 79,439 & HS23/36 & B & $1.5 \pm 0.41$ \\
\hline G1 & 79,444 & HS23/36 & C & $1.6 \pm 0.49$ \\
\hline G1 & 79,443 & $\mathrm{HS} 4$ & B & $1.70 \pm 0.53$ \\
\hline G1 & 79,253 & HS23/36 & C & $1.02 \pm 0.02$ \\
\hline G1 & 79,429 & HS19 & B & $1.34 \pm 0.29$ \\
\hline G1 & 79,440 & HS23/36 & B & $3.68 \pm 1.3$ \\
\hline \multirow[t]{2}{*}{ G1 } & 79,421 & HS2 & C & $2.65 \pm 0.99$ \\
\hline & & & & Total: Mean of means: $2.2 \pm 0.71$ \\
\hline G2 & 79,441 & HS23/36 & - & $1.1 \pm 0.12$ \\
\hline \multirow[t]{2}{*}{ G2 } & 79,428 & HS19 & - & $1.21 \pm 0.19$ \\
\hline & & & & Total: Mean of means: $1.15 \pm 0.15$ \\
\hline G3 & 79,442 & - & C & $1.64 \pm 0.45$ \\
\hline Reference & 79,436 & - & - & $1 \pm 0$ \\
\hline
\end{tabular}

G1: Isolates with capsular genotype and LOS class

G2: Isolates with capsular genotype and without LOS class

G3: Isolates without capsular genotype and with LOS class

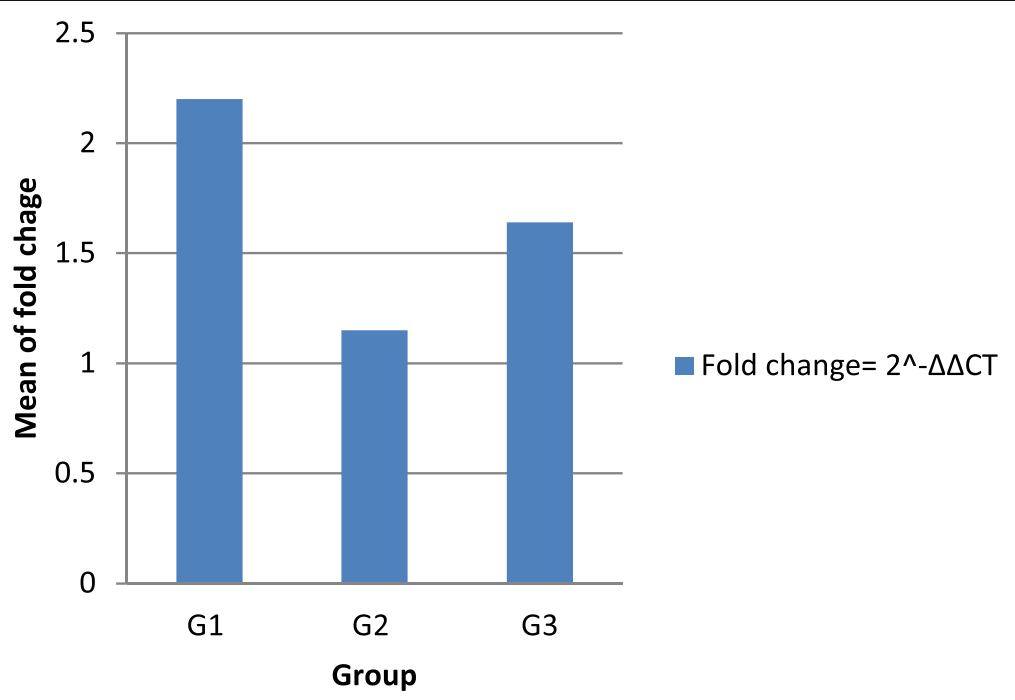

Fig. 2 Level of dnaK gene expression in clinical C. jejuni isolatesof different groups 


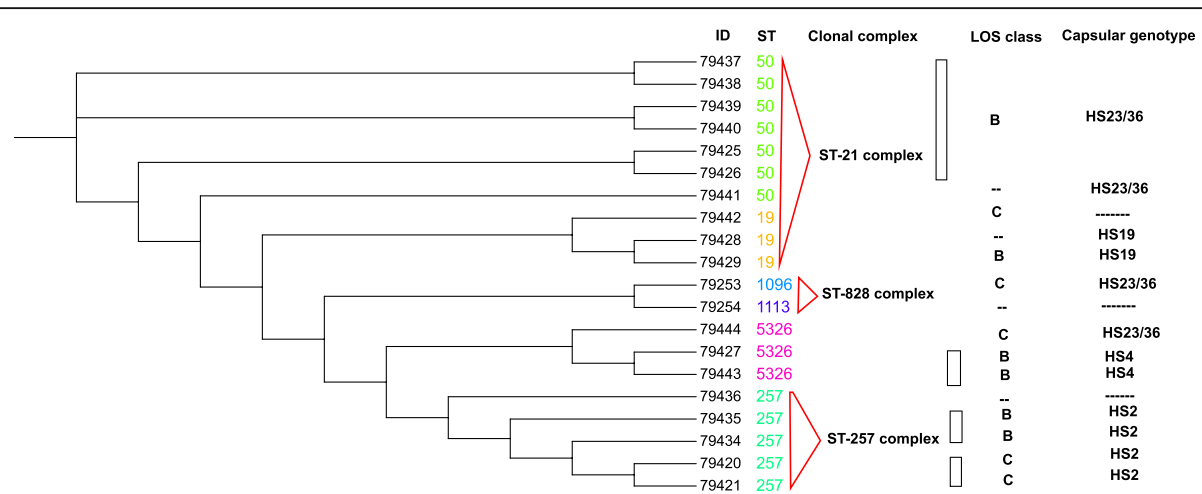

Fig. 3 Dendrogram demonstrating the phylogenetic relationship between the 20 C. jejuni isolates from patients with gastroenteritis in Iran 2018. *Dendrogran plotted by Interactive Tree of Life (iTOL) v4 free access mode [19]

World map related to C. jejuni strains from 1980 to 2018 shows the distribution of ST19 (CC21), ST50 (CC21), and ST257 (CC257) among different continents (Figs. 8, 9 and 10). The frequency of CC21 strains was associated to sporadic cases of human gastroenteritis which was recorded from America (USA and Canada), Europe (UK, The Netherlands and Germany), Asia (Iran, this study) and Australia. Inclusive comparison of CC257 (including ST-257) strains in the same time period showed recorded strains from Asia (Iran, this study), Europe (UK and Spain), America (Chile) and Africa (South Africa).

In a dendrogram constructed by inclusive comparison of MLST results during 2014-2018 a similar picture was depicted for distribution of CC21 and CC257 clonal complexes. Moreover, neighbor-joining results indicated that $\mathrm{CC} 21$ and CC353 complexes are the most divers, most frequent and most widely distributed clonal complexes around the world, respectively; although, CC353 was not detected in the current study (Fig. 11) (Table 6).

\section{Discussion}

Campylobacter spp. is considered to be the most common bacterial cause of human gastroenteritis in the world [21]. We aimed to investigate CPS types, sialylated LOS classes and MLST types of virulent $C$. jejuni strains isolated from children with gastroenteritis aged 0-5 years in Tehran, Iran.

Collection time of our isolates was between May and October. Epidemiological reports from other countries $[22,23]$ suggest that human campylobacteriosis tends to increase during this period of year.

Epidemiological investigations have shown that a $C$. jejuni infection precedes GBS in 20 to $50 \%$ of cases in Europe, North and South America, Japan, and Australia [23]. Sialylated LOS loci of A, B and C classes as well as HS types of HS2, HS4, HS23/36c and HS19 are accused to be associated with GBS patients $[2,23,24]$. The prevalence of LOS class B was $(11 / 20 ; 55 \%)$, followed by class C $(5 / 20 ; 25 \%)$, while class A was not detected. Serichantalergs et al., also reported the sialylated LOS

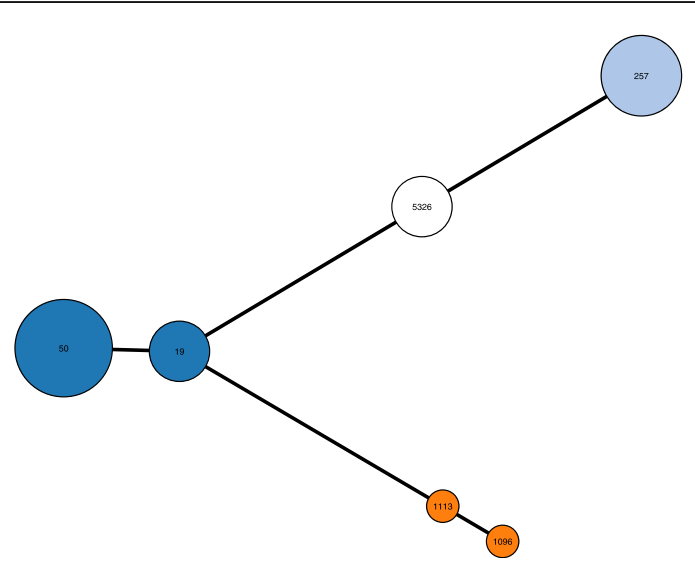

clonal complex (MLST)

ST-21 complex [10]

mplex [5]

ST-828 complex [2]

Fig. 4 Minimum spanning tree for categorical data (based on clonal complexes) The tree was created using GrapeTree [20]. Each clonal complex is represented by a circle, numbers in each circle related to STs. the number of isolates is shown in brackets 


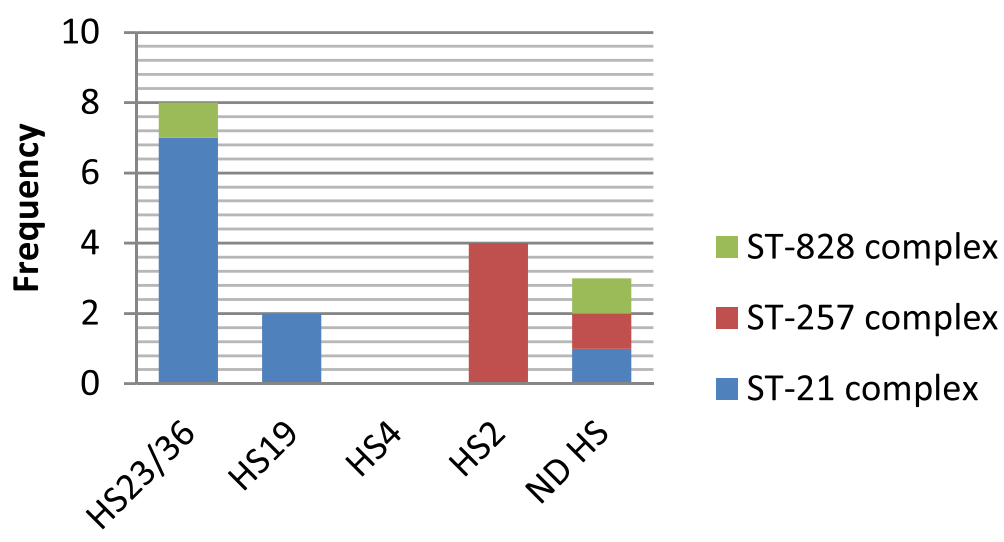

Capsular type

Fig. 5 Linkage between CPS genotypes and MLST CC

classes A, B and C in children with gastroenteritis which is consistent with our results [25].

Dominant CPS genotype among our isolates was HS23/36c (9/20) followed by HS2 (4/20). In an overall picture depicted by Pike et al., these two genotypes, after HS4, comprise the most prevalent CPS types worldwide [26]. Consistent with our findings, Sainato et al. identified HS2 and HS4 as two of eight most prevalent CPS types in pediatric population with gastroenteritis in Egypt [27]. Two CPS types of HS1 and HS41 were not detected among our $20 \mathrm{C}$. jejuni isolates. But, according to the report of Pike et al., HS1 was one of the dominant CPS types in the overall picture [26]. This may be due to the limited period and small number of our isolates compared with isolates from various populations and in a longer time period.

A wide range of CPS types including HS1/44, HS2, HS4, HS19 and HS23/36c are usually identified in C. jejuni strains isolated from GBS patients [2, 24]. In our gastroenteritis-related samples $85 \%$ of strains expressed one of HS23/36c, HS2, HS4 and HS19, which signifies the high probability of GBS progress in this group of patients.

In Iran, studies reported non-polio acute flaccid paralysis (AFP) incidence rates ranging from 0.3 to 6.5 per 100,000 [28]. The annual incidence of GBS is $0.6-4$ cases per 100,000 populations that is a most common cause of nono-polio worldwide. Also in Iran, GBS is the most common cause of paralysis among AFP patients [29-31]. Almost 25-40\% of GBS patients worldwide suffer from $C$. jejuni infection 1-3 weeks prior to the illness [32]. We demonstrated that $C$. jejuni isolates had the predominant LOS class B and C, and capsular genotypes of HS23/36, HS2, HS4 and HS19, all of which are accused to be associated with the progress of gastroenteritis toward GBS. Therefore, attention to incidence of $C$. jejuni, together with proper identification and treatment of children with campylobacteriosis is necessary for prevention of subsequent GBS.

Our findings showed that the dnaK expression mean in strains with specified capsular genotype and sialylated

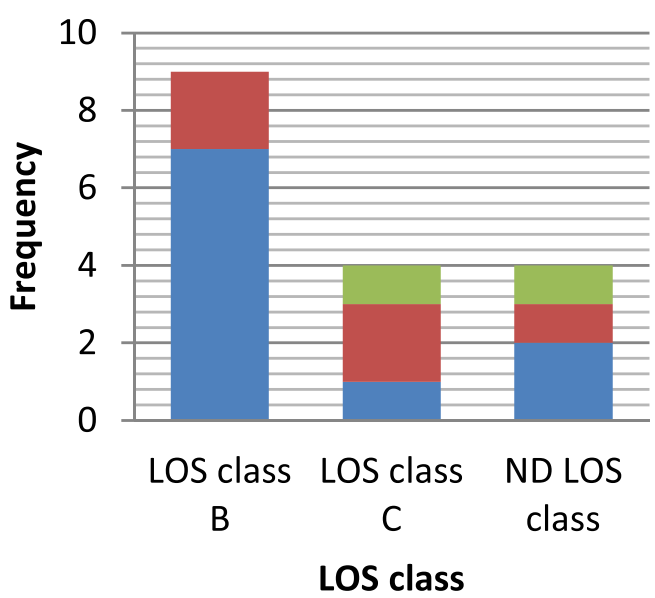

ST-828 complex

ST-257 complex

ST-21 complex

Fig. 6 Linkage between LOS class and MLST CC 


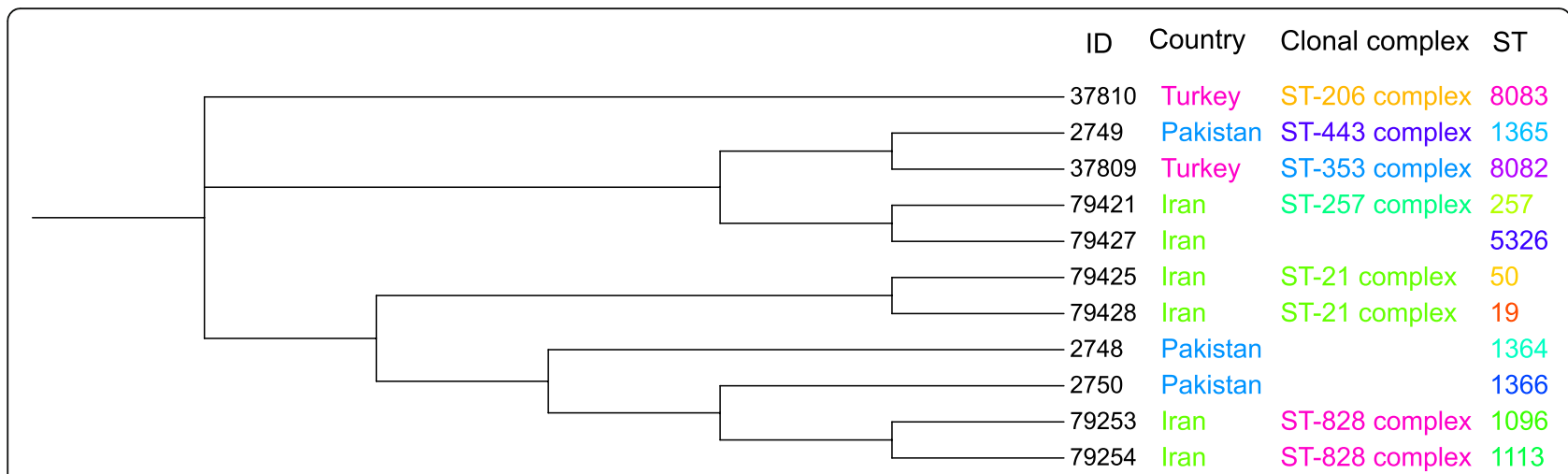

Fig. 7 Phylogenetic analysis of 11 C. jejuni strains for Iran, Turkey and Pakistan. *Dendrogran plotted by Interactive Tree of Life (iTOL) v4 free access mode [19]

LOS was greater than that of others (with either capsular genotype or LOS class). Furthermore, our finding revealed that the expression of $d n a K$ was higher when sialylated LOS and particular capsular genotypes simultaneously are present in a strain. Moreover, it was shown by $\mathrm{HU}$ et al., that dnaK gene expression is upregulated in conditions simulating in-vivo which means it may be induced in infected human host. Considering the crucial role of dnaK in antigenic mimicry and GBS, it can be concluded that individuals infected with $C$. jejuni strains having sialylated LOS classes and the selected capsular serotypes as well as a high expression profile of dnaK may be more likely to develop GBS. Furthermore,
dnaK gene can be mentioned as an antigen candidate in preventive studies or as a diagnostic marker [10].

Genetic variations in $20 \mathrm{C}$. jejuni strains from enteritis patients was also identified by MLST. A total of 6 STs were observed and $17 / 20$ (85\%) belonged to 3 clonal complexes (CCs), while 3 isolates belonged to STs unassigned to a CC.

The majority of $C$. jejuni strains were assigned to CC21; this finding supports previous observations which shows CC21 is the most prevalent CC worldwide [33]. Within CC21, ST-50 was the dominant ST in our clinical samples, although not all of previous studies reported ST-50 as the dominant sequence type $[34,35]$.

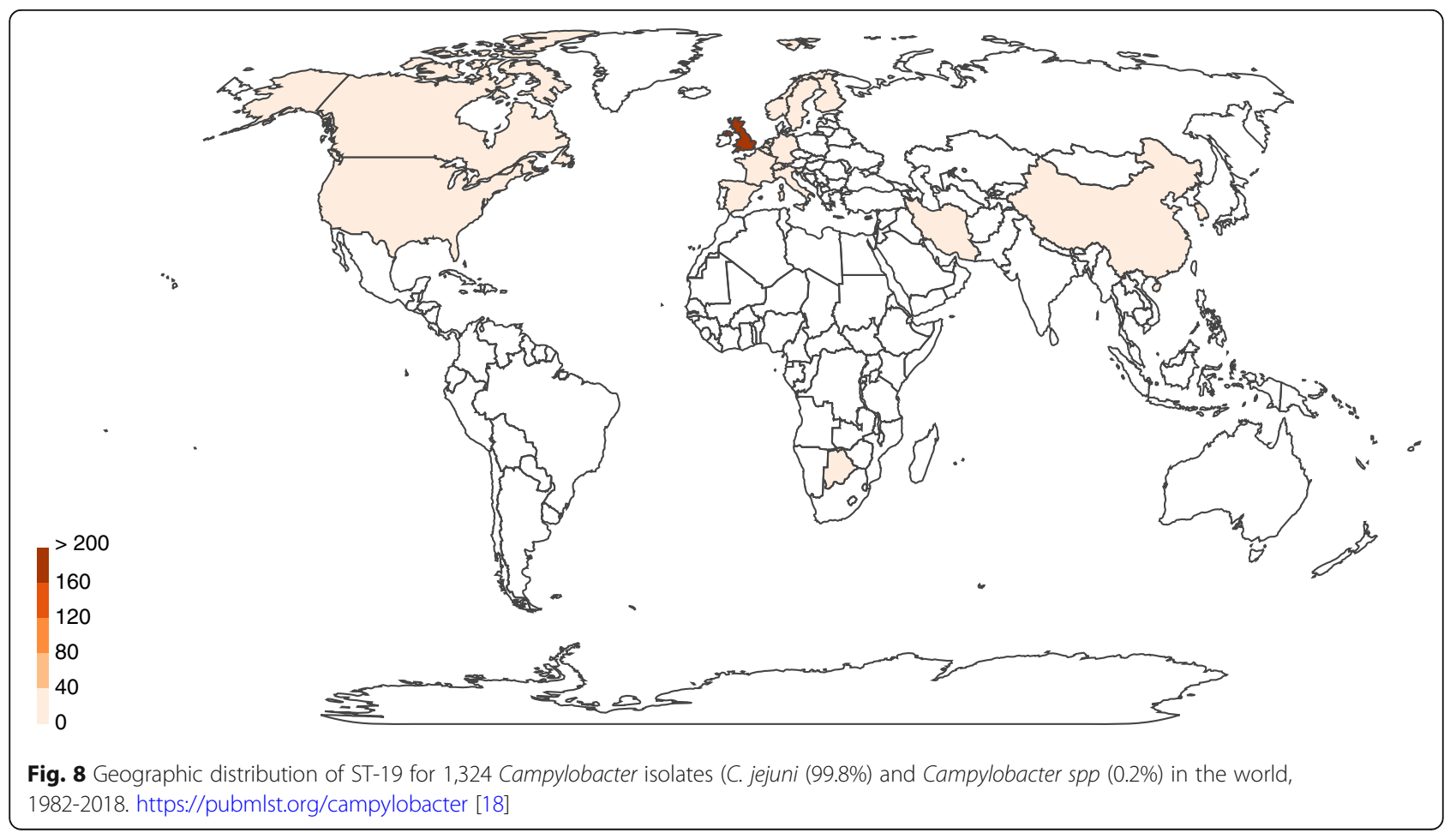



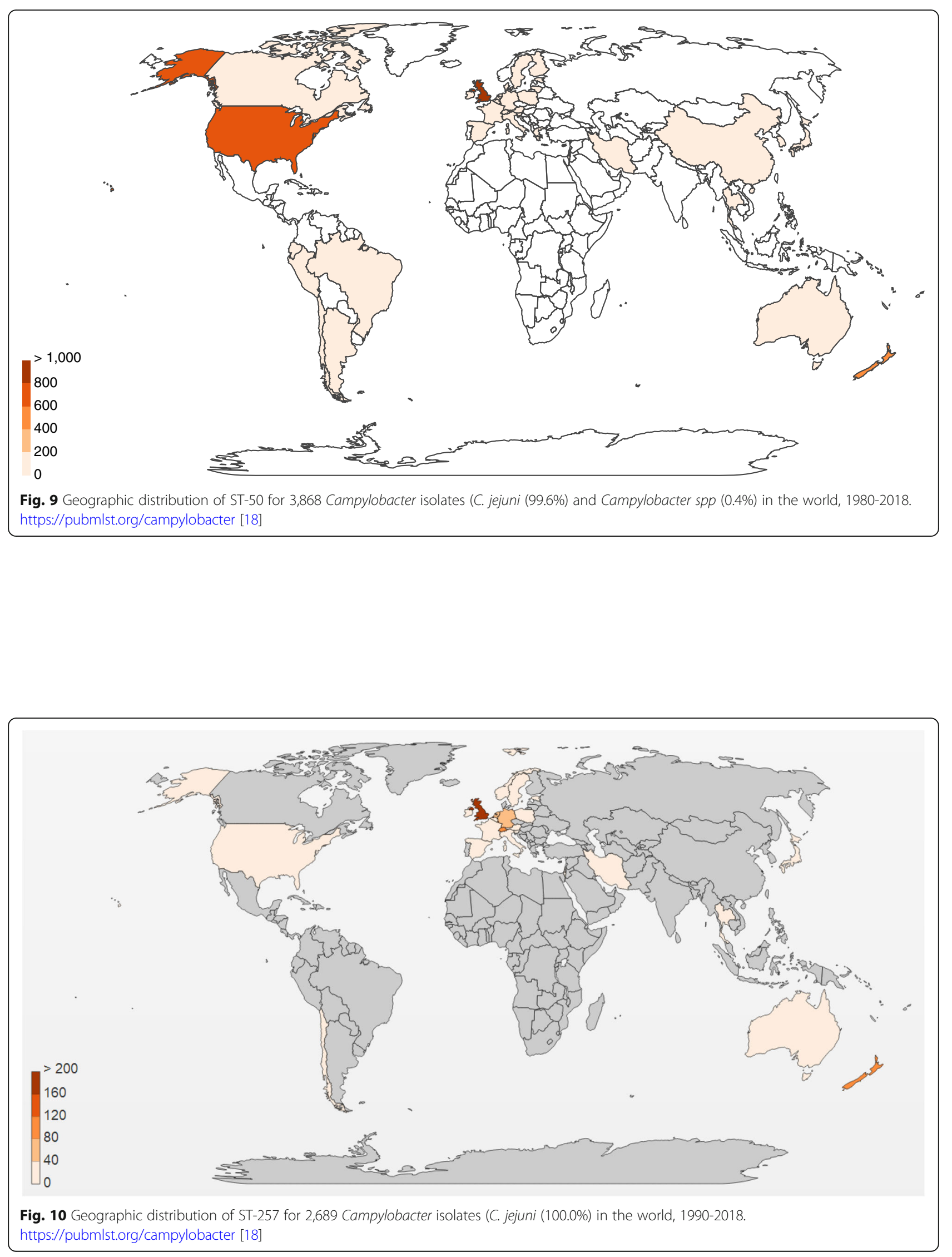


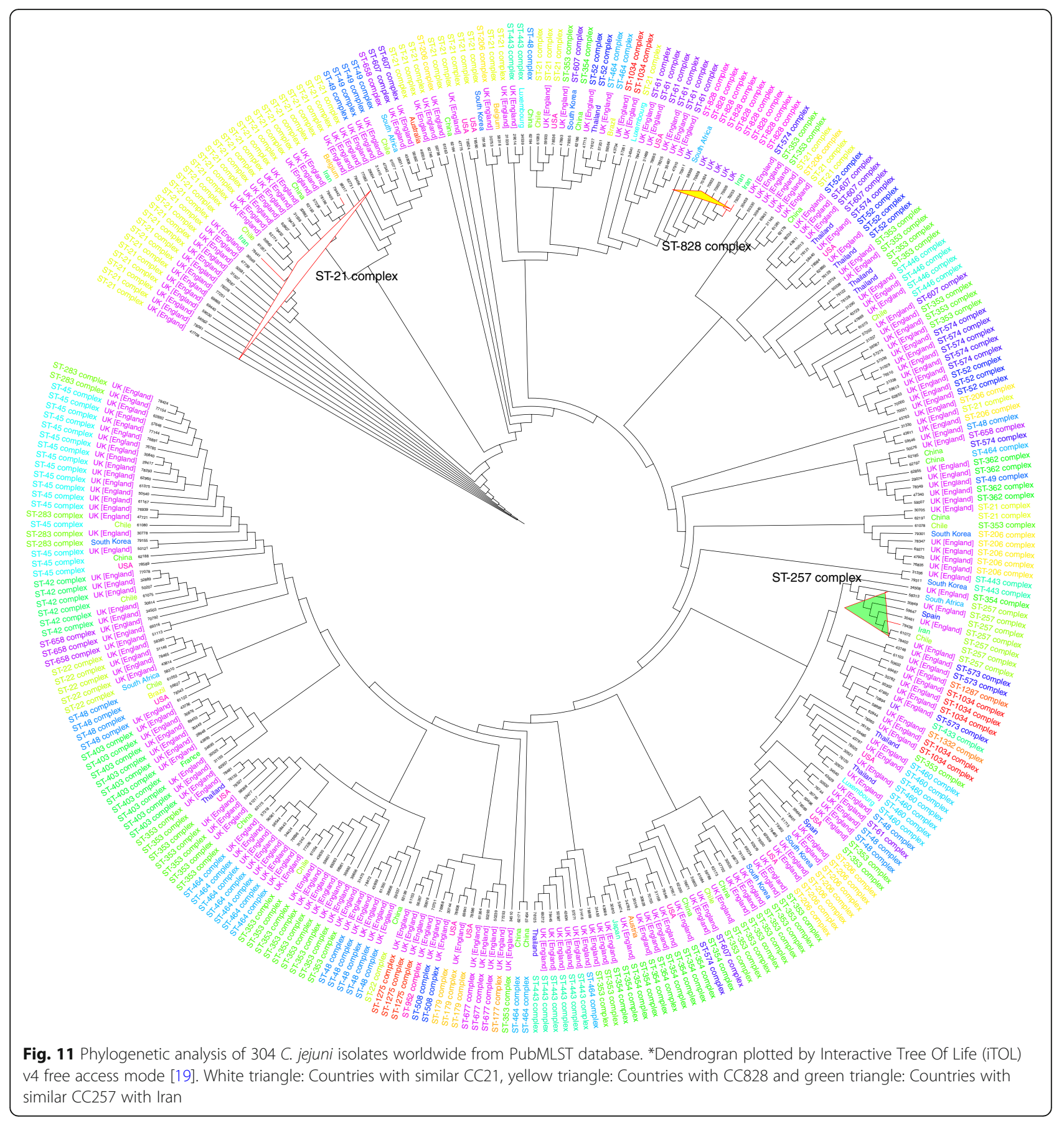

Meanwhile, consistent with our finding, ST-50 and ST-19 (CC21) and ST-257 were mostly related to human campylobacteriosis cases [36-38]. However, isolates from other sources (fresh whole retail chicken, raw milk and environmental water) also presented CC21 as dominant CCs [39, 40].

The correlation between certain MLST clonal complexes and LOS classes and HS types were investigated in present study. The majority of $C$. jejuni isolates in ST-21 $(7 / 10 ; 70 \%)$ expressed LOS class B, while both LOS class B and C occurred in ST-257 complex in an equal percentage $(2 / 5 ; 40 \%)$. Habib et al., demonstrated that ST-21 complex strongly correlated with LOS class C [23] but this combination was rare among our isolates.

MLST analysis demonstrated that ST-21 and ST-257 complexes were dominants in our enteritis $C$. jejuni strains. Overall of different studies, the ST-22 complex was significantly overrepresented in the GBS isolates and ST-21 complex in enteritis isolates [2, 24, 35]. No new 
Table 6 Top 10 Clonal complexes based on frequency and diversity extracted from circle dendrogram

\begin{tabular}{|c|c|c|c|c|c|}
\hline Rank & Clonal Complex & Diversity & Rank & Clonal Complex & Frequency \\
\hline 1 & ST-353 Complex & 12 & 1 & ST-21 Complex & 51 \\
\hline 2 & ST-21 Complex & 10 & 2 & ST-353 Complex & 42 \\
\hline 3 & ST-206 Complex & 7 & 3 & ST-206 Complex & 17 \\
\hline 4 & ST-48 Complex & 6 & 4 & ST-45 Complex & 17 \\
\hline 5 & ST-464 Complex & 5 & 5 & ST-48 Complex & 14 \\
\hline 6 & ST-52 Complex & 5 & 6 & ST-354 Complex & 13 \\
\hline 7 & ST-574 Complex & 5 & 7 & ST-464 Complex & 13 \\
\hline 8 & ST-607 Complex & 5 & 8 & ST-403 Complex & 10 \\
\hline 9 & ST-354 Complex & 4 & 9 & ST-443 Complex & 10 \\
\hline 10 & ST-1034 Complex & 3 & 10 & ST-52 Complex & 9 \\
\hline
\end{tabular}

sequence type was detected in present study. Moreover, among neighbor countries, only a few data from Pakistan and Turkey was available and phylogenetic analysis revealed no common ST or CC with Iran.

Inclusive comparison of MLST results demonstrated that ST-50 (CC21) was widely distributed in different countries including UK, USA, Canada, some European countries, Australia and China, while ST-19 (CC21) and ST-257 (CC257) was less ubiquitously spread and absent from Australia and China/Canada, respectively.

Moreover, neighbor-joining results indicated that CC21 and CC353 are the most diverse, most frequent and most widely distributed clonal complex around the world; although, CC353 was not detected in current study. The most diverse CCs are related to more prevalent sequence types. This proposes that probably their diversity is a mirror of their replication frequency and circulation which affects their gene content and efficiency. This shows the movement of $C$. jejuni strains beyond the boundaries. The occurrence of identical clonal complexes with different capsular types and LOS classes is consistent with genetic variation in circulating identical genotypes and their evolution toward different pathotypes probably through acquisition of different genetic elements including LOS and CPS gene clusters.

\section{Conclusions}

To our knowledge, this is the first report identifying CPS types and sialylated LOS classes, as well as MLST genotyping of $C$. jejuni strains related to gastroenteritis of children in Tehran, Iran. We demonstrated that i) C. jejuni isolates had the predominant LOS class B and C, and capsular genotypes of HS23/36, HS2, HS4 and HS19, all of which are accused to be associated with the progress of disease toward GBS; ii) Higher expression level of dnaK gene was detected in strains with HS23/36c, HS2 and HS4 capsular genotypes and sialylated LOS classes B or C, therefore its expression can be used as an indicator for probable GBS progress in infected patients; iii) Isolates were highly genetically diverse and distributed in 6 STs. CC21 and CC257 were predominant in our isolates. Comprehensive comparison of MLST results demonstrated that CC21 is the largest clonal complex of $C$. jejuni strains worldwide and provides a universal picture of movement of dominant Campylobacter strains.

\section{Abbreviations \\ C. jejuni: Campylobacter jejuni; CPS: Capsular Polysaccharide; HS: Heat-Stable; GBS: Guillain-Barre syndrome; LOS: Lipo-Oligosaccharide; HSP: Heat Shock Proteins; MLST: Multilocus sequence typing; mCCDA: modified charcoal- cefoperazone-deoxycholate agar; ST: Sequence Types; CC: Clonal Complexes;} iTOL: Interactive Tree of Life

\section{Acknowledgements}

The current article is a part of the first author's PhD thesis carried out in Tarbiat Modares University. The authors would like to thank Dr. Astrid P. Heikema from the Erasmus Medical Center, Rotterdam, for providing positive controls of CPS genotypes and LOS locus classes.

For figures no. 4, 8, 9 and 10, this publication made use of the PubMLST website (https://pubmlst.org/) developed by Keith Jolley (Jolley et al. Wellcome Open Res 2018, 3:124 [version 1; referees: 2 approved]) and sited at the University of Oxford. The development of that website was funded by the Wellcome Trust. Hereby we declare the use of iTOL free access mode in producing figures no. 3, 7 and 11 .

\section{Authors' contributions}

Mahnaz Sarhangi collected all specimens and performed all the laboratory tests, also had a major contribution in drafting the main manuscript and prepared all figures and tables and had approved the submitted version. Bita Bakhshi designed and supervised the study entirely and had a major contribution in writing the manuscript and had approved the submitted version. Shahin Najar Peeraeyeh had has a major role in analysis of the data as well as drafting the main manuscript and had approved the submitted version. The authors read and approved the final manuscript.

\section{Funding}

None to declare.

\section{Availability of data and materials}

The datasets of the current study are available within article or can be obtained from corresponding upon request. The Accession Numbers of DNA sequences of 20 C. jejuni isolates were deposited in GenBank at https:// pubmlst.org/bigsdb?db=pubmlst_campylobacter_isolates\&page=query. The values are as follows: 79420, 79421, 79425, 79426, 79427, 79428, 79429, 79434, 79435, 79436, 79437, 79438, 79439, 79440, 79441, 79442, 79443, 79444,79253 and 79254.

\section{Ethics approval and consent to participate}

The study was reviewed and approved by the Medical Ethics Committee of Tarbiat Modares University (Code: IR.TMU.REC.1396.587) before it began and 
all research was performed in accordance with relevant guidelines/ regulations. The clinical specimens were obtained from Microbiology Laboratory of 3 children hospitals, Tehran, Iran. The consent to participate was obtained from the parents/guardians of the minors included in this study and the data were analyzed anonymously.

\section{Consent for publication}

Not applicable.

\section{Competing interests}

The authors declare that they have no competing interests or personal relationships that could have influenced the work reported in this paper.

Received: 29 March 2020 Accepted: 7 January 2021

Published online: 23 January 2021

\section{References}

1. Habib I, De Zutter L, Uyttendaele M. Eleven campylobacter species. Food Microbiol. 2019:263-87.

2. Heikema AP, Islam Z, Horst-Kreft D, Huizinga R, Jacobs BC, Wagenaar JA, et al. Campylobacter jejuni capsular genotypes are related to Guillain-Barré syndrome. Clin Microbiol Infect. 2015;21(9):852. e1-9.

3. Poly F, Serichatalergs $\mathrm{O}$, Schulman M, Ju J, Cates CN, Kanipes M, et al. Discrimination of major capsular types of campylobacter jejuni by multiplex PCR. J Clin Microbiol. 2011:49(5):1750-7.

4. Liang H, Zhang A, Gu Y, You Y, Zhang J, Zhang M. Genetic characteristics and multiple-PCR development for capsular identification of specific serotypes of campylobacter jejuni. PLoS One. 2016;11(10):e0165159.

5. Poly F, Serichantalergs O, Kuroiwa J, Pootong P, Mason C, Guerry P, et al. Updated campylobacter jejuni capsule PCR multiplex typing system and its application to clinical isolates from south and Southeast Asia. PLoS One. 2015;10(12):e0144349.

6. Parker CT, Horn ST, Gilbert M, Miller WG, Woodward DL, Mandrell RE. Comparison of campylobacter jejuni lipooligosaccharide biosynthesis loci from a variety of sources. J Clin Microbiol. 2005:43(6):2771-81.

7. Hameed A, Woodacre A, Machado LR, Marsden GL. An updated classification system and review of the lipooligosaccharide biosynthesis gene locus in campylobacter jejuni. Front Microbiol. 2020;11:677.

8. Godschalk PC, Heikema AP, Gilbert M, Komagamine T, Ang CW, Glerum J, et al. The crucial role of campylobacter jejuni genes in anti-ganglioside antibody induction in Guillain-Barre syndrome. J Clin Invest. 2004;1 14(11):1659-65.

9. Loshaj-Shala A, Regazzoni L, Daci A, Orioli M, Brezovska K, Panovska AP, et al. Guillain Barré syndrome (GBS): new insights in the molecular mimicry between C. jejuni and human peripheral nerve (HPN) proteins. J Neuroimmunol. 2015;289:168-76.

10. Hu Y, Shang $Y$, Huang J, Wang $Y$, Ren F, Jiao $Y$, et al. A novel immunoproteomics method for identifying in vivo-induced Campylobacter jejuni antigens using preadsorbed sera from infected patients. Biochim Biophysic Acta. 2013;1830(11):522935.

11. Yonekura K, Yokota S-I, Tanaka S, Kubota H, Fujii N, Matsumoto H, et al. Prevalence of anti-heat shock protein antibodies in cerebrospinal fluids of patients with Guillain-Barré syndrome. J Neuroimmunol. 2004;156(1-2):204-9.

12. Murphy C, Carroll C, Jordan K. Environmental survival mechanisms of the foodborne pathogen campylobacter jejuni. J Appl Microbiol. 2006;100(4):623-32.

13. Shams S, Bakhshi B, Moghadam TT. In Silico analysis of the cadF gene and development of a duplex polymerase chain reaction for species-specific identification of campylobacter jejuni and campylobacter coli. Jundishapur J Microbiol. 2016;9(2):e29645.

14. Loshaj-Shala A, Panovska AP, Brezovska K, Beretta G, Suturkova L, Apostolski S. Involvement of serum HSP 70 in Guillain-Barré syndrome: an exploratory study and a review of current literature. Maced Pharma Bull. 2015;61(1):61-7.

15. Helgeland G, Petzold A, Hoff JM, Gilhus NE, Plant GT, Romi FR. Anti-heat shock protein 70 antibody levels are increased in myasthenia gravis and Guillain-Barré syndrome. J Neuroimmunol. 2010;225(1-2):180-3.

16. Bronnec $V$, Turoňová $H$, Bouju $A$, Cruveiller $S$, Rodrigues $R$, Demnerova $K$, et al. Adhesion, biofilm formation, and genomic features of campylobacter jejuni bf, an atypical strain able to grow under aerobic conditions. Front Microbiol. 2016;7:1002.

17. Dingle K, Colles F, Wareing D, Ure R, Fox A, Bolton F, et al. Multilocus sequence typing system forCampylobacter jejuni. J Clin Microbiol. 2001;39(1):14-23.

18. Jolley KA, Bray JE, Maiden MC. Open-access bacterial population genomics: BIGSdb software, the PubMLST. org website and their applications. Wellcome Open Res. 2018;3:124.
19. Letunic I, Bork P. Interactive tree of life (iTOL) v4: recent updates and new developments. Nucleic Acids Res. 2019;47(W1):W256-W9.

20. Zhou Z, Alikhan N-F, Sergeant MJ, Luhmann N, Vaz C, Francisco AP, et al. GrapeTree: visualization of core genomic relationships among 100,000 bacterial pathogens. Genome Res. 2018;28(9):1395-404.

21. Cody AJ, Maiden MC, Strachan NJ, McCarthy ND. A systematic review of source attribution of human campylobacteriosis using multilocus sequence typing. Eurosurveillance. 2019;24(43):1800696.

22. Naumova EN, Jagai JS, Matyas B, DeMaria A, MacNeill I, Griffiths J. Seasonality in six enterically transmitted diseases and ambient temperature. Epidemiol Infect. 2007; 135(2):281-92.

23. Habib I, Louwen R, Uyttendaele M, Houf $K$, Vandenberg O, Nieuwenhuis EE, et al. Correlation between genotypic diversity, lipooligosaccharide gene locus class variation, and caco-2 cell invasion potential of campylobacter jejuni isolates from chicken meat and humans: contribution to virulotyping. Appl Environ Microbiol. 2009;75(13):4277-88

24. Islam Z, van Belkum A, Wagenaar JA, Cody AJ, de Boer AG, Tabor H, et al. Comparative genotyping of campylobacter jejuni strains from patients with GuillainBarré syndrome in Bangladesh. PLoS One. 2009;4(9):e7257.

25. Serichantalergs $O$, Wassanarungroj P, Khemnu N, Poly F, Guerry P, Bodhidatta L, et al. Distribution of genes related to type 6 secretion system and lipooligosaccharide that induced ganglioside mimicry among campylobacter jejuni isolated from human diarrhea in Thailand. Gut Pathog. 2020;12:1-10.

26. Pike BL, Guerry P, Poly F. Global distribution of campylobacter jejuni Penner serotypes: a systematic review. PLoS One. 2013;8(6):e67375.

27. Sainato R, ElGendy A, Poly F, Kuroiwa J, Guerry P, Riddle MS, et al. Epidemiology of campylobacter infections among children in Egypt. Am J Trop Med Hyg. 2018;98(2): $581-5$.

28. Momen AA, Shakurnia A. An epidemiological analysis of acute flaccid paralysis in Khuzestan Province, Southwest Iran, from 2006 to 2010. Epidemiol Health. 2016;38: e2016030.

29. Momen AA, Shakurnia A, Momen M. Eleven-year surveillance of acute flaccid paralysis in southwestern Iran. Turk J Pediatr. 2019;61(4):544-51.

30. Naeini AE, Ghazavi M, Moghim S, Sabaghi A, Fadaei R. Acute flaccid paralysis surveillance: a 6 years study, Isfahan, Iran. Adv Biomed Res. 2015:4:99.

31. Soltani J, Esmailnasab N, Roshani D, Karimi M, Amjadi M. Acute flaccid paralysis and its differential diagnosis in in kurdistan province, Western Iran; an 11-year surveillance. Iran J Pediatr. 2014;24(2):131.

32. Nyati KK, Nyati R. Role of campylobacter jejuni infection in the pathogenesis of Guillain-Barré syndrome: an update. Biomed Res Int. 2013;2013:852195.

33. Zhang P, Zhang X, Liu Y, Jiang J, Shen Z, Chen Q, et al. Multilocus Sequence Types and Antimicrobial Resistance of Campylobacter jejuni and C. coli Isolates of Human Patients From Beijing, China, 2017-2018. Front Microbiol. 2020;11:2542.

34. Piccirillo A, Giacomelli M, Salata C, Bettanello S, De Canale E, Palù G. Multilocus sequence typing of campylobacter jejuni and campylobacter coli from humans and chickens in north-eastern Italy. New Microbiol. 2014;37(4):557-62.

35. Nielsen LN, Sheppard S, MCCarthy N, Maiden M, Ingmer H, Krogfelt K. MLST clustering of campylobacter jejuni isolates from patients with gastroenteritis, reactive arthritis and Guillain-Barré syndrome. J Appl Microbiol. 2010;108(2):591-9.

36. Aksomaitiene J, Ramonaite S, Tamuleviciene E, Novoslavskij A, Alter T, Malakauskas M. Overlap of antibiotic resistant campylobacter jejuni MLST genotypes isolated from humans, broiler products, dairy cattle and wild birds in Lithuania. Front Microbiol. 2019;10:1377.

37. Harvala H, Rosendal T, Lahti E, Engvall EO, Brytting M, Wallensten A, et al. Epidemiology of Campylobacter jejuni infections in Sweden, November 2011October 2012: is the severity of infection associated with C. jejuni sequence type? Infect Ecol Epidemiol. 2016;6(1):31079.

38. Wieczorek K, Wołkowicz T, Osek J. MLST-based genetic relatedness of campylobacter jejuni isolated from chickens and humans in Poland. PLoS One. 2020;15(1):e0226238.

39. Lévesque S, Frost E, Arbeit RD, Michaud S. Multilocus sequence typing of campylobacter jejuni isolates from humans, chickens, raw milk, and environmental water in Quebec, Canada. J Clin Microbiol. 2008;46(10):3404-11.

40. Kovanen S, Rossi M, Pohja-Mykrä M, Nieminen T, Raunio-Saarnisto M, Sauvala M, et al. Population genetics and characterization of Campylobacter jejuni isolates from western jackdaws and game birds in Finland. Appl Environ Microbiol. 2019;85(4): e02365-18.

\section{Publisher's Note}

Springer Nature remains neutral with regard to jurisdictional claims in published maps and institutional affiliations. 